\title{
Expert Opinion: A Call for Basal Insulin Titration in Patients with Type 2 Diabetes in Daily Practice: Southeast European Perspective
}

\author{
Nicolae Hancu • Andrej Janez · Nebojsa Lalic · Nikolaos Papanas • \\ Dario Rahelic • Gabriela Roman • Cristian Serafinceanu • \\ Nikolaos Tentolouris · Blaženko Vukovic · Agron Ylli • \\ Tsvetalina Tankova
}

Received: January 29, 2021 / Accepted: February 17, 2021 / Published online: March 15, 2021

(C) The Author(s) 2021

\section{ABSTRACT}

Therapeutic inertia related to insulin treatment, i.e. delays in initiation, especially titration of basal insulin, is a significant problem in daily

\section{N. Hancu (ه)}

Regina Maria Clinic, University of Medicine and Pharmacy Iuliu Hatieganu, Cluj Napoca, Romania e-mail: nhancu@umfcluj.ro

\section{A. Janez}

Department of Endocrinology, Diabetes and Metabolic Diseases, University Clinical Center Ljubljana, Ljubljana, Slovenia

\section{N. Lalic}

Clinic for Endocrinology, Diabetes and Metabolic Diseases, Clinical Center of Serbia, Faculty of Medicine, University of Belgrade, Belgrade, Serbia

\section{N. Papanas}

Diabetes Centre, Second Department of Internal Medicine, Medical School, Democritus University of Thrace, University Hospital of Alexandroupolis, Alexandroupolis, Greece

\section{Rahelic}

Vuk Vrhovac University Clinic for Diabetes, Endocrinology and Metabolic Diseases, Merkur University Hospital, Zagreb, Croatia

\section{Rahelic}

University of Zagreb School of Medicine, Zagreb, Croatia

D. Rahelic

Josip Juraj Strossmayer, University of Osijek School of Medicine, Osijek, Croatia practice in Southeast European countries. This phenomenon can be traced back to several patient-, physician- and health system-related factors. In recognition of the issue of inadequate insulin titration, 11 leading experts from countries in this region held a consensus-

\section{G. Roman}

Clinical Centre of Diabetes, Nutrition and Metabolic

Diseases, Emergency Clinic County Hospital,

University of Medicine and Pharmacy Iuliu

Hatieganu, Cluj-Napoca, Romania

C. Serafinceanu

Diabetology Clinic National Institute of Diabetes, Nutrition and Metabolic Diseases Prof. NC Paulescu, Carol Davila University of Medicine and Pharmacy, Bucharest, Romania

N. Tentolouris

Laiko General Hospital, National and Kapodistrian, University of Athens, Medical School, Athens, Greece

B. Vukovic

Department of Endocrinology With General Internal Medicine, University Clinical Center of Republika Srpska, Banja Luka, Bosnia and Herzegovina

A. Ylli

Endocrinology Service in UHC Mother Teresa Tirane, Tirane, Albania

T. Tankova

Department of Endocrinology, Faculty of Medicine, Medical University of Sofia, Sofia, Bulgaria 
seeking meeting to review the current status of insulin initiation after non-insulin treatment and the potential barriers to insulin titration to provide an algorithm and tools for outpatient physicians and for patients aimed at optimizing basal insulin titration. The experts reached a consensus on the majority of the topics and proposed recommendations on how clinical inertia can be overcome. The outcomes of the meeting have been summarized in this paper.

Keywords: Clinical inertia; Insulin initiation; Insulin titration; Type 2 diabetes

\section{Key Summary Points}

Therapeutic inertia, especially in titration of basal insulin, can be traced back to several patient-, physician- and health system-related factors, and is a significant problem in daily practice in Southeast European countries.

Leading experts from Southeast European countries held a consensus-seeking meeting to review the current status of insulin initiation after non-insulin treatment and the potential barriers to insulin titration.

The experts provided outpatient physicians with the detailed steps of insulin initiation and the patient characteristics that should be considered when choosing injectable therapies.

The experts provided a basal insulin titration guide for the patients that help basal insulin titration.

The experts reached consensus on several aspects of basal insulin initiation but did not reach definite consensus on keeping versus discontinuing sulfonylurea treatment after insulin initiation.

\section{DIGITAL FEATURES}

This article is published with digital features, including a summary slide, to facilitate understanding of the article. To view digital features for this article go to https://doi.org/10.6084/ m9.figshare.14039354.

\section{INTRODUCTION}

As a result of the progressive nature of type 2 diabetes (T2D), antihyperglycaemic treatment needs to be intensified in most cases after firstline monotherapy with metformin. Basal insulin can be an option after metformin monotherapy or part of treatment intensification beyond two medications [1]. Although glucagon-like peptide 1 receptor agonists (GLP1 RAs) have been recommended by international guidelines as first injectable, basal insulin should be chosen in the presence of certain patient characteristics. Once basal insulin is introduced, its dose should be titrated on the basis of a target fasting glucose level using an evidence-based titration algorithm.

Therapeutic or clinical inertia is defined as the "failure to intensify therapy when treatment targets are not met" [1]. Inertia related to basal insulin treatment might occur because of a delay in initiation or inadequate titration [2]. Both patients and healthcare professionals have an essential role in diabetes management, so inertia is primarily the responsibility of these stakeholders [2]. Patients may fear hypoglycaemia, weight gain, increased treatment complexity and injection pain or may feel selfblame or failure. Physicians may lack confidence in their patients' ability to administer daily injections and manage the complex process of self-titration and may have concerns about the additional time required for patient education $[3,4]$. In addition, health or drug reimbursement systems might represent inertia factors due to a fragmented healthcare system [1] or due to the costs of new drugs [2, 5].

Delays in treatment intensification can further increase the risk of macrovascular events and microvascular complications stemming from inadequate glycaemic control $[2,6]$. An 
extensive systematic literature review [7] indicated that time to treatment intensification ranged from 0.3 to more than 7.2 years (with a median value of more than 1 year), and the time during which glycated haemoglobin A1 (HbA1c) levels were above the study specific threshold ranged from 0.4 to more than 7.2 years. On the other hand, delayed deintensification of a therapeutic regimen can also be an important inertia factor, as overtreatment conveys a risk of further complications, especially in the elderly population [8].

In addition, the large observational Diabetes Management Practice Study found that less than one-third of patients with T2D and onefifth of patients on insulin treatment achieved an HbA1c target range of below 7\% [9]. Surveys $[10,11]$ have also demonstrated significant insulin initiation inertia in Central and Southeast European countries.

Inadequate insulin titration is also a frequent clinical inertia factor. The observational DUNE study demonstrated a $22 \%$ achievement rate for individualized HbA1c targets [12]. Longer-term real-world data of patients newly switched to basal insulin analogues demonstrated that the great majority of patients remained uncontrolled 6 months after insulin initiation [13]. Inadequate titration was also observed in randomized studies of insulin glargine $300 \mathrm{U} / \mathrm{mL}$ (Gla-300) [14]. The randomized controlled Take Control study provided evidence that self-titration is more effective in achieving glycaemic control than physician-managed titration [15].

A Canadian expert panel that provided a detailed practical approach to insulin therapy in patients with T2D [16] clearly stated that "insulin initiation and titration are two separate behaviours for patients" and that physicians should address both when managing patients.

The advantages of insulin analogues over human insulin for hypoglycaemia have been widely demonstrated [17]. The advantageous effects of second-generation basal insulin analogues (Gla-300 and insulin degludec) on the risk of overall and nocturnal hypoglycaemia have been underlined in recent recommendations $[1,16]$.

There are expert recommendations $[16,18]$ on insulin initiation and titration, including basal insulin initial dose, fasting plasma glucose (FPG) target range, dose adjustment algorithms, optimal insulin dose at the end of the titration process, use of other antihyperglycaemic drugs after initiating basal insulin, managing insulin distress, performing motivational interviews, or conversing with patients about insulin initiation after a diabetes diagnosis.

Recognizing the general issue of inadequate insulin titration in the Southeast region of Europe and taking into account the advantages of the newest-generation basal insulins in terms of hypoglycaemia and weight gain, a meeting was organized with the participation of leading experts from Southeast European (SEE) countries with the following aims:

(1) To review insulin initiation after insufficiency of dual or triple non-insulin treatment

(2) To review the current status and potential barriers of insulin titration

(3) To develop an algorithm and tools for outpatient physicians and patients aimed at optimizing basal insulin (BI) titration

\section{METHODS}

The expert kick-off meeting was held on 29 May 2020 (as an online meeting).

The meeting began by reviewing the current daily practice of insulin initiation and titration in the participating experts' countries, including the implementation and adaptation of the international and national guidelines, the therapeutic position of insulin and other injectable agents, and the aspects of daily practice that should be revised. At the end of the online meeting, the experts opened the discussion for three workshops on the following topics:

- Key principles when initiating and titrating basal insulin-based therapy

- Patient involvement in diabetes management

- Next steps for patients not optimally controlled on a basal insulin-based regimen 
The experts had 3 weeks to share their opinions, answer the raised questions, and send feedback to the other experts. Sanofi provided an online application for the discussion.

After closing the online discussion, a draft report was prepared and circulated among the experts for review and comments. At the final stage, another online meeting was arranged for some remaining open questions to be discussed and clarified. The final version of the report was approved by experts on 3 August 2020 .

This article is based on previously conducted studies and does not contain any new studies with human participants or animals performed by any of the authors.

\section{RESULTS}

\section{Key Principles When Initiating Basal Insulin-Based Therapy}

The experts first reviewed the current recommendations, the general daily practice in the Southeast European region and patient characteristics and other factors that should be considered when selecting from different treatment regimens. The experts agreed that inadequate titration as a type of clinical inertia plays a significant role in suboptimal glycaemic control in a relatively large proportion of patients in the region.

\section{Factors That Determine Inertia in Basal Insulin Analogue (BIA) Initiation \& Titration (There Are Factors in Addition to Titration)}

The experts identified many clinical inertia factors and made proposals that could potentially overcome these barriers. These factors and approaches are summarized in Table 1.

\section{Patient Characteristics That Should Be Considered When Choosing Between Basal Insulin, GLP-1 RA or an FRC (Fixed Ratio Combination)}

According to the latest American Diabetes Association/European Association for the Study of Diabetes (ADA/EASD) Consensus report, if glycaemic control is inadequate with dual or triple oral therapy, the first injectable drug or drug combination should be considered. When choosing between them, glycaemic status (including the primary reason for high HbA1c levels-fasting or postprandial hyperglycaemia - and the presence of signs and symptoms of a catabolic state), the risk of hypoglycaemia, obesity and comorbidities should be taken into account. Basal insulin is preferred as first injectable when HbA1c > 11\% or there is evidence of catabolism and thereafter as an intensifying option if the patient does not reach glycaemic targets with OADs/GLP-1RA. When choosing insulin, it should be considered that it is the most potent antihyperglycaemic agent and has beneficial effects on triglyceride and HDL cholesterol levels.

GLP-1 RAs are indicated when:

- HbA1c is above the target but $<10 \%$ after dual/triple therapy.

- Body weight needs to be reduced.

- There is increased risk of hypo events.

- The patient's self-monitoring capability is limited.

- The patient has established atherosclerotic disease (GLP-1 RA with proven benefit).

FRC of GLP-1 RA + basal insulin is recommended when (also see "Recommended Steps When Initiating FRC"):

- HbA1c is $>10 \%$ and the patient is obese.

- There is an increased risk of hypo.

- FPG is close to the target, but postprandial blood glucose (PPG) and HbA1c are above the target despite optimally titrated BIA.

\section{Key Principles That Should Be Followed When} Physicians Initiate and Titrate BIA

The experts defined six steps from patient selection to stopping titration that physicians should consider when initiating and titrating a BIA.

STEP I-Preparing for initiation

A. Selecting the right patients for (BIA) treatment

When deciding whether a patient should initiate basal insulin treatment, several general and diabetes-specific medical history data and patient characteristics should be evaluated. 
Table 1 Clinical inertia factors and potential approaches to overcome them

\section{Clinical inertia factors}

BI inertia factors on the patient's side

Fear of

Disease progression-insulin considered "the last stage of his/her disease"

Hypoglycaemia (and inability to control night hypo events)

Weight gain

Other side effects

Self-monitoring/self-injection

Feeling self-blame for failure in treating diabetes

Aversion to

Regular blood glucose monitoring

Complexity of titration

Inability to perform self-titration

BI inertia factors on the physician's side

(The physician's role in inertia is more important than the patient's role)

Feelings of uncertainty about how effective titration might be

Insufficient knowledge and/or commitment

Lack of

Time to explain

Time to educate

Knowledge about the differences between the newer BIAs and the older BIs

Experience in insulin treatment

Educators in some countries in the region

Reluctance regarding the need for frequent visits

Fear of side effects of insulin treatment (hypos and weight gain)

Concern about the patients' willingness to titrate and perform SMBG

Misunderstanding the recommendations and guidelines, i.e. SGLT2i and/or GLP-1 RA should be initiated early in high CV/renal risk patients independently of $\mathrm{HbAlc}$ levels

\section{Potential ways to overcome}

Potential approaches to overcome inertia on the patient's side

Adequate education of the patient on the benefits of insulin therapy and the importance of titration

Good communication between the patient and the physician, creating an atmosphere of confidence

Educating the patient to perform blood glucose selfmonitoring

Ensuring availability and easy access to a GP/endocrinologist Involving a psychologist and a well-trained nurse on the team when initiating insulin

Providing the patient with simple titration steps and offering the option of phone consultation with his/her physician

Potential approaches to overcome inertia on the physician's side

Continuous education of healthcare professionals at all health system levels

To be aware of the differences between the newer BIAs and the older BIs

To be aware that modern BIA and BI/GLP-1RA FRCs are safe and convenient for the patients

To be aware of the phenomenon of inertia in BIA initiation and titration

To improve general knowledge about the current treatment approaches in the guidelines

To improve the quality of titration

Training and involvement of nurse educators in diabetes management

$B I / B I A$ basal insulin/basal insulin analogue, $C V$ cardiovascular, GLP-1 RA glucagon-like peptide 1 receptor agonist, $H b A 1 c$ haemoglobin A1c, $S G L T 2 i$ sodium-glucose cotransporter 2 inhibitor, $S M B G$ self-monitoring of blood glucose, $F R C$ fixed ratio combination 
These are the most important characteristics of patients who may need BIA treatment:

- Newly diagnosed T2D that is symptomatic with $\mathrm{HbA} 1 \mathrm{c} \geq 9 \%$

- T2D that cannot be controlled (i.e. target HbA1c is not reached) with maximally tolerated doses of non-insulin agents

- Diabetic ketoacidosis (DKA) and/or hyperglycaemic hyperosmolar state

- End-stage organ failure (e.g. kidney failure, hepatic illness)

- Acute illness (e.g. COVID-19 disease)

- Gestational diabetes

- Intolerance to oral medications

- Patient preference

- When the physician considers BIA the most appropriate approach

However, the following characteristics indicate that a patient should not be treated with insulin:

- In those of advanced age when patients may not be able to manage self-injection and/or perform blood glucose self-monitoring

- In those with short life expectancy

- In those at high risk for hypoglycaemia

B. Individualized HbA1c targets

HbA1c targets should be determined individually on the basis of guidelines, recommendations and individual clinical data, such as duration of diabetes, existing complications and comorbidities, frequency and severity of hypoglycaemic events, and patient life expectancy.

C. Helping the patient cope with challenges

To cope with challenges related to BI-based treatment, the physician, in collaboration with a diabetes educator or nurse, has to inform the patient why he/she needs BIA, what the metabolic aims are with the newly initiated BI and the expected benefits of an insulin analogue compared to human insulin. The physician should educate the patient on how to perform self-injection, to monitor blood glucose levels, to self-titrate insulin doses and to integrate these steps into his/her daily life. As part of these preparatory steps, the physician should provide the patient with written (web-based) informational material, demonstrate a positive attitude and obtain patients' consent for cooperation.

D. Making decisions on the type of insulin

After completing the steps outlined above, the type of BIA initiated, the time of application (e.g. bedtime), and the details of self-monitoring and self-titration should be defined.

STEP II-Initiation of BIA: setting FPG targets and training on SMBG (self-monitoring of blood glucose) for titration

The aims of the following technical description of insulin initiation are to diminish the potential risks associated with BIA initiation and to improve efficacy of the first steps. When initiating BI, the initial dose, the method of selfmonitoring, and the period of time for the initial dose should be determined.

- The initial dose should be set to $10 \mathrm{U}$ or $0.1-0.2 \mathrm{U} / \mathrm{kg}$ body weight (morning/midday/evening, according to patient preference and lifestyle).

- The patient should be trained on how and when to self-monitor FPG

- (a) For example, every 3 days or

- (b) On the same days of the week (e.g. on Mondays and Thursdays) or

- (c) According to a selected algorithm

If any option from the above is selected, the pattern of self-monitoring should be clearly explained to the patient.

- The first injection should be administered in the physician's office.

- The patient should be advised to continue the initial dose until the first phone call with his/her treating physician, educator or diabetes nurse before beginning titration.

STEP III-Titration until reaching the target FPG

The aim of titration is to achieve optimal glycaemic control by gradually increasing the daily insulin dose. Titration should also be individualized on the basis of individual glycaemic targets and other patient characteristics. The overall titration process covers the first contact with the patient after initiation, the process of dose increase and monitoring, including FPG level change and detection of hypoglycaemic events, as well as scheduled 
communication between the patient and the physician.

There are several steps in insulin titration:

- First call

- At the end of the first week

- With the physician, educator or diabetes nurse

- Self-titration

- After the first phone call with the physician

- $2 \mathrm{U}$ dose increase every 3 days to reach FPG target without hypos

- In case of hypo

- The cause of hypo should be determined

- If no clear reason, the dose should be reduced by $10 \%$

- Repeated call during the second week

- Titration of insulin until target FPG reached

- Communication: the patient together with his/her physician should determine the mode of communication

- The patient should schedule a visit with his/ her physician 1 month after insulin initiation

STEP IV-Stop titration

Titration can lead to three potential outcomes that indicate titration should be stopped.

Target FPG is reached

Titration should be stopped when target FPG is reached, and the last dose should be continued when the target FPG has been reached without hypoglycaemic events. Self-monitoring of blood glucose should be continued every 3 days or on the same days of the week (e.g. on Mondays and Thursdays), and HbA1c should be checked after 3 months.

The patient experiences hypo events

Titration should be stopped when the patient experiences hypo events. Hypo events should be verified (i.e. plasma glucose $<70 \mathrm{mg}$ / $\mathrm{dL}[<3.9 \mathrm{mmol} / \mathrm{L}])$. Potential errors in insulin administration, diet, alcohol consumption, physical activity (including sexual activity) should be checked and corrected if needed. In case hypo is corrected, titration should be continued; if not, the insulin dose should be reduced by $10-20 \%$.

HbA1c target cannot be reached

When the HbA1c target cannot be reached with BIA doses of $50-60 \mathrm{U} /$ day or $0.7-1 \mathrm{U} / \mathrm{kg}$ body weight/day after 3 months, regardless of whether FPG is in the target range, the physician should

- Carefully review the patient's lifestyle and insulin injection technique and adherence to treatment

- Consider treatment intensification

In addition to providing patients with all necessary information on the initiation and titration of BIA, in countries where general practitioners are involved in insulin treatment, they should also be educated on how to effectively manage their patients' switching to BIA, including SMBG and dose titration.

Steps I to IV are summarized in Fig. 1.

\section{Expert Opinion on the Continuation/ Discontinuation of Sulfonylureas and Other Oral Antihyperglycaemic Agents}

The experts fully agreed that metformin should be continued with initiation of BIA unless contraindications or intolerance prevent its use.

On the other hand, there was no full agreement on the continuation of sulfonylureas (SUs) when BIA was initiated. The experts stated that SUs are widely used in countries in the SEE region, even after insulin initiation. Although they acknowledged that SUs may have beneficial effects (e.g. on glucose toxicity), they underlined the risk of hypoglycaemic events and high glucose variability when continuing SUs. They highlighted that patients in SEE countries are frequently treated by general practitioners who are not necessarily familiar with the placement of different SU generations in diabetes management. Thus, most experts agreed that SUs should be discontinued or replaced with safer drugs when an injectable is administered, especially when GLP-1 RAs and modern BIAs are available. However, some experts would allow their use at lower doses until initiation of prandial insulin. Moreover, they highlighted that C-peptide level, even if not routinely measured in the outpatient 


\section{Steps to be followed when BIA is initiated}
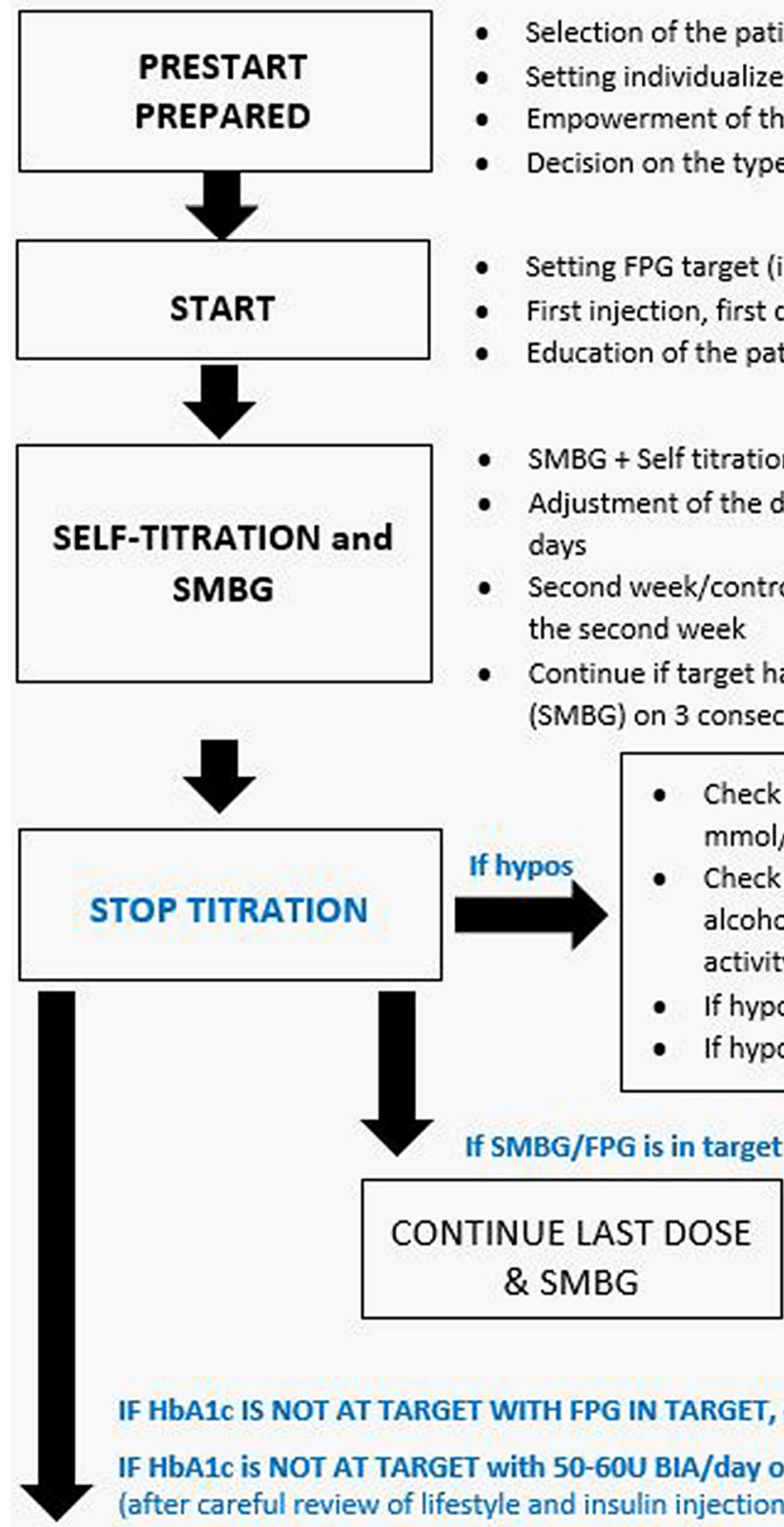

- Selection of the patients

- Setting individualized HbA1c target

- Empowerment of the patient

- Decision on the type of basal insulin

- Setting FPG target (in line with HbAlc target)

- First injection, first dose

- Education of the patient on SMBG

- $S M B G+$ Self titration - call with the patient at the end of the first week

- Adjustment of the dose based on mean FPG (SMBG) on 3 consecutive days

- Second week/control/continue? - call with the patient at the end of the second week

- Continue if target has been reached based on mean/intermediate FPG (SMBG) on 3 consecutive days

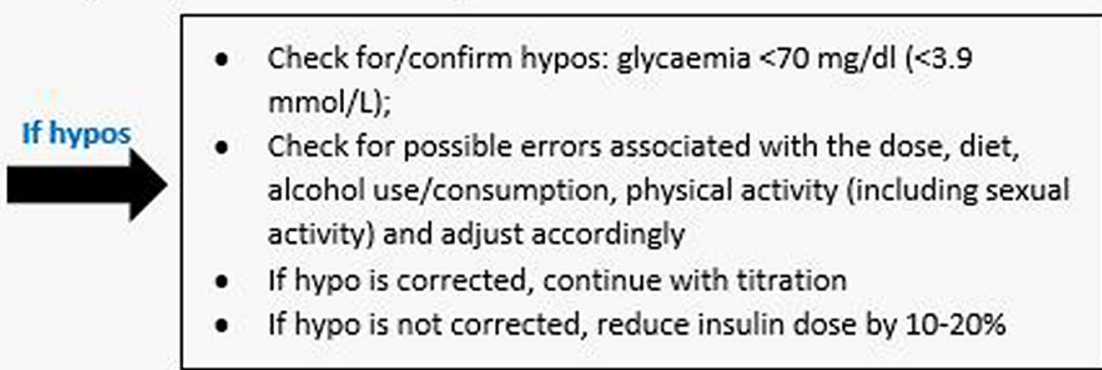

If SMBG/FPG is in target without hypos

- SMBG on 3 consecutive days

- HbAlc at 3 months

- Adjustment of the dose when needed

IF HbA1C IS NOT AT TARGET WITH FPG IN TARGET, or

IF HbA1c is NOT AT TARGET with $50-60 \mathrm{U} \mathrm{BIA} /$ day or $0.7-1 \mathrm{U} / \mathrm{kg}$ body weight after 3-6 months (after careful review of lifestyle and insulin injection technique)

\section{INTENSIFICATION}

BIA: basal insulin/basal insulin analogue; FPG: fasting blood glucose; HbA1c: haemoglobin A1c; SMBG: self-monitoring of blood glucose

Fig. 1 Summary of steps I-IV 
setting, may play a role in this decision, as very low C-peptide levels indicate that there is no place for SUs.

\section{Recommended Steps When Initiating the Basal Plus or Basal Bolus Regimen}

The experts recommended two options for adding prandial insulins (PIs) to a BIA-based regimen:

- Option 1. Stepwise addition of PI: starting at mealtime with the highest postprandial (PP) glycaemia, followed by the second and third meals with the highest PP glycaemia

- Option 2. Introduction of the full basal bolus (BB) regimen from the beginning

Of these, option 1, "stepwise addition" is the recommended approach.

With either option, the physician should ensure that the patient is capable and willing to comply with the requirements of the new regimen. The patient should be provided with information about the new regimen and the new insulin type, as well as the new monitoring and titration schedule that the new treatment regimen requires with special attention to the timing of the meal covered by PI. Moreover, for option 1, the patient should perform a "7point" glycaemic profile to identify the meal with the highest PP glycaemia, as PI should be initiated at the meal with the highest PP glycaemia or with the largest meal. Blood glucose levels should be monitored, and the doses should be titrated accordingly.

Overall, short-acting insulin analogues are preferred to human insulins.

\section{Recommended Steps When Initiating FRC}

The characteristics of patients for whom FRC seems to be the best choice of treatment are listed in Sect. "Patient Characteristics That Should Be Considered When Choosing Between Basal Insulin, GLP-1 RA or an FRC (Fixed Ratio Combination)". In addition, the physician should ask the patient about his/her personal or family history of medullary thyroid carcinoma (because of a general warning when GLP-1 RAs are used) and should provide the patient with a written titration algorithm and information about FRCs. Titration of FRCs should respect the same principles as BIA initiation, being as simple as possible, and should be performed weekly to avoid gastrointestinal side effects or every 3 days with regular follow-up, especially during the first 2-3 months.

FRC should be administered at the same time every day and can be aligned to the main meal. As FRCs are relatively new products on the market, HCPs/family physicians may need education.

\section{How to Involve the Patient in Diabetes Management}

\section{Steps and Elements of the Individualized Approach}

The individualized approach is essential so that physicians can adapt all steps of BIA initiation and titration to each patient's requirements and lifestyle. The individualized approach requires that the physician become acquainted with the patient's daily life and identify his/her requirements and difficulties, with the aim of obtaining the patient's confidence and reassuring him/her about the safety of BIAs.

The individualized approach should focus on the following domains:

- Individualized contact schedule with the patient

- Individualized glycaemic targets

- Individualized titration process

The information provided to the patients should be adapted to the patient's level of knowledge, education, mental status, manual dexterity, social status, motivation and family support.

\section{Patient Guide for Basal Insulin Titration}

Taking into account that the initiation and titration of insulin is an important step in the course of diabetes, physicians should make great efforts to explore and answer all questions raised by the patient. The experts agreed to prepare a patient guide on insulin titration with the steps of the titration algorithm.

Figure 2 summarizes this consensus patient guide with a short introductory description and a list of parameters with the titration algorithm. 


\section{Patient Basal Insulin Titration Guide}

Type 2 diabetes (T2D) is a progressive disease. At the time of diagnosis, more than $50 \%$ of $\beta$-cells are already lost and continue to decline. Therefore, the use of insulin is an appropriate option at any point in the management of T2D to replace the insulin that the pancreas is unable to sufficiently produce. Common side effects of Insulin therapy are low blood glucose ("hypoglycaemia") and weight gain. The effect of insulin depends on food intake, physical activity, and alcohol consumption.

International guidelines recommend initiation of insulin with basal insulin, primarily with basal insulin analogues, followed by titration to a target fasting glucose. Insulin titration, the process of insulin dose adjustment based on the measured fasting glucose values, is critical in helping patients with T2D to reach their glycaemic goals.

\begin{tabular}{|c|c|}
\hline Parameter & Detailed description \\
\hline Individualized glycaemic targets & 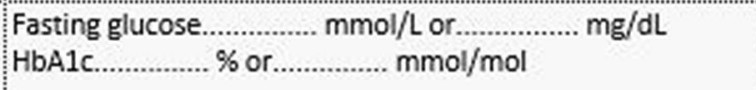 \\
\hline Selected basal insulin/analogue & .................... (name) \\
\hline Starting dose & ..................... U/day \\
\hline Time of administration? & 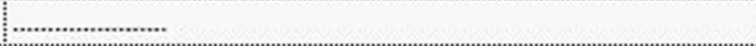 \\
\hline $\begin{array}{l}\text { Fasting glucose for basal insulin dose } \\
\text { adjustment }\end{array}$ & $\begin{array}{l}\text { Measure fasting glucose .................. (when) } \\
\text { Fasting glucose value (measure on } 3 \text { consecutive days and } \\
\text { take the mean value or intermediate value based on } \\
\text { physician discretion) }\end{array}$ \\
\hline $\begin{array}{l}\text { Frequency of basal insulin dose } \\
\text { adjustment }\end{array}$ & Every............... days \\
\hline $\begin{array}{l}\text { Basal insulin dose step increase/decrease } \\
\text { The dose should be incrementally } \\
\text { increased on a regular basis using target } \\
\text { FPG }\end{array}$ & 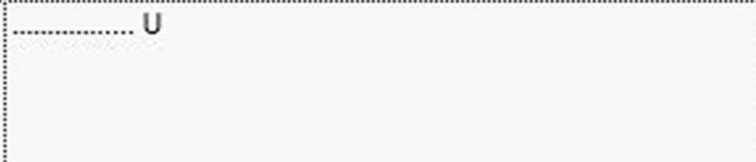 \\
\hline $\begin{array}{l}\text { Basal insulin titration scheme } \\
\text { Ranges of Fasting glucose for } \\
\text { increase/maintenance/decrease of BI dose } \\
\text { with the defined dose step }\end{array}$ & 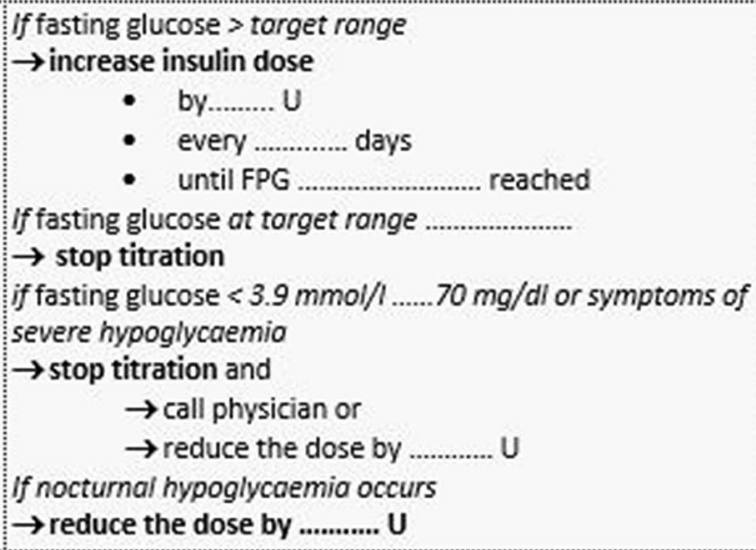 \\
\hline $\begin{array}{l}\text { Call physician regularly } \\
\text { after measuring Fasting glucose } 3 \\
\text { consecutive days }\end{array}$ & $\begin{array}{l}\text { Every week during the first ....... weeks } \\
\text { In case of hypoglycaemia }\end{array}$ \\
\hline \multicolumn{2}{|l|}{ HbA1c: haemoglobin A1c; T2D: type 2 diabetes } \\
\hline
\end{tabular}

Fig. 2 Consensus guide for the patients 
The treating physician should fill in the table with patient-specific values.

\section{Additional Patient Guide Elements for Basal Bolus and FRC Treatment}

In addition to the patient guide elements for BI treatment initiation and titration, there are elements that need to be considered when prandial insulin is added to the regimen or when an FRC is initiated.

Additional elements of the patient guide when adding prandial insulin to basal insulin:

- Setting a PPG target

- Instructions on the selected SMBG scheme adapted to the $\mathrm{BP} / \mathrm{BB}$ regimen

- Titration adapted to $\mathrm{BP} / \mathrm{BB}$ regimen

- Self-adjustment of insulin doses (both basal and prandial)

- How to adjust to physical activities

- How to adjust to caloric intake

- Weight gain issues/nutrition advice

Additional elements of the patient guide when initiating an FRC:

- Short explanation to the patient on

- The rationale of using FRC

- The metabolic targets

- Specific side effects

- How FRCs should be administered

- How FRCs should be titrated

- How self-monitoring should be adapted to FRC

\section{Next Steps for Patients Not Optimally Controlled on a Basal Insulin-Based Regimen}

\section{Checklist of Parameters That Can Indicate the Need for Further BI Titration or Intensification}

The experts prepared a list of parameters that an outpatient physician should check to determine the next steps for a patient treated with basal insulin who is inadequately controlled or has frequent hypoglycaemic events. In addition, the experts listed the steps and specified the reevaluation frequency.

Parameters that should be checked:

- Basic glycaemic parameters

- HbA1c

- Fasting or pre-prandial plasma glucose

- PPG

- Daily glucose profile

- Other patient characteristics

- Patient medical history

- Overall treatment history

- GI side effects during previous GLP-1 RA treatment

- Family history of medullary thyroid carcinoma

- Hypoglycaemic events

- Frequency and severity (overall and nocturnal)

- Symptomatic and not symptomatic

- Body weight

- Diabetes duration

- Existing comorbidities (including renal status), especially in cases of titration, switching or intensification

- Global cardiovascular risk and diseases

- C-peptide levels when measured in outpatient setting

- Patient preferences and lifestyle (diet, physical activity, etc.)

Recommended frequency of re-evaluation:

- At the beginning-up to a month-weekly

- Upon stabilization at 3-month intervals

\section{Proposed Next Steps Based on Patient Profile}

The experts summarized the clinical situations that outpatient physicians should consider when they plan to undertake BI titration, switch to BIA or enact treatment intensification.

HbA1c and FPG are high without experiencing hypoglycaemic events:

- BI doses should be titrated

HbA1c is high and the patient is overweight: 
- Adding a GLP-1 RA to BI (or FRCs) should be considered

HbA1c and PPG are high:

- Basal-bolus regimen or

- Combined BIA + GLP-1 RA should be considered

\section{If hypoglycaemic events occur:}

- Doses of basal insulin should be adjusted

In addition, some experts recommended the use of SGLT2 (sodium-glucose cotransporter 2) inhibitors in the case of postprandial hyperglycaemia with FPG levels within the target, also taking into account evidence on the beneficial effects of SGLT2 inhibitors, which was underlined in the latest ADA/EASD position paper.

If titration of BIA is needed, it should be performed without delay. Any treatment modification should be discussed with the patients so that his/her adherence is optimal.

\section{DISCUSSION}

The progressive nature of type 2 diabetes usually necessitates continuous intensification of therapy in order to reach glycaemic control. Accordingly, when dual or triple oral regimens fail to control the glycaemic status, injectable therapies should be initiated, of which GLP-1 RAs are recommended. However, there are patients for whom basal insulin or basal insulin plus GLP1-RA fixed ratio combinations are the best choice.

Timely insulin initiation and titration to a glycaemic target are key factors in achieving adequate glycaemic control, and any clinical inertia may have adverse physiological consequences. Although there are many potential ways to overcome both patient and physician factors that lead to inertia, inertia in initiation, especially titration of insulin, is a major problem. Potential activities that can diminish clinical inertia include education of and communication with the patients, providing personalized patient guides and simple algorithms. The advantages of insulin analogues over human insulin-especially the lower risk of overall and nocturnal hypoglycaemia-may also help the patients and physicians overcome clinical inertia.

As clinical inertia is a specific major problem in the Southeast European (SEE) region, Sanofi organized a consensus-seeking meeting with the following aims: (1) to review the current status of insulin initiation and titration, (2) to review therapeutic options after failure of dual or triple noninsulin treatment, and (3) to provide an algorithm and tools for outpatient physicians and for patients to optimize basal insulin (BI) titration.

Eleven leading experts from eight countries in the SEE region were invited to participate. They approved the proposed topics before the meeting.

The experts reached consensus on (1) the primary factors contributing to clinical inertia and proposed steps to overcome identified barriers; (2) a simple algorithm that physicians should follow when selecting the most appropriate injectable therapy; (3) the key principles and practical steps that should be followed when initiating basal insulin analogue-based regimens combined or not with prandial insulin or GLP1-1 RAs; (4) patient management that meets the requirement of the principle of a personalized approach; and (5) a patient guide aimed to help patients with basal insulin initiation and titration steps. A question in which no definite consensus was reached was whether to keep or discontinue sulfonylureas after initiation of insulin.

Similar to other expert consensuses, these recommendations do not substitute or overwrite currently available international and national guidelines or rules that provide guides for patient management and define the application of diagnostic and treatment steps, such as country-specific reimbursement rules for some drugs. The primary aims were to assist in the routine everyday clinical work of physicians who treat patients with type 2 diabetes and to improve management of these patients.

\section{CONCLUSIONS}

Acknowledging the major problem of clinical inertia in SEE countries in basal insulin 
regimen-treated patients led experts from these countries to propose recommendations on how clinical inertia can be overcome, reaching a consensus on all but one discussed topic. The experts call for a systematic approach in basal insulin titration and propose guides to be followed by both the treating physicians and patients to increase the probability of reaching the glycaemic goal in a real-life setting in a safe manner.

\section{ACKNOWLEDGEMENTS}

Funding. Sanofi Bulgaria EOOD, SanofiAventis groupe S.A Albania, Sanofi Aventis Groupe Representative Office Bosnia and Herzegovina, sanofi-aventis Croatia d.o.o., Sanofi Aventis d.o.o. Ljubljana Slovenia, Sanofi Aventis AEBE Greece, S.C. Sanofi Romania SRL, and Sanofi-Aventis DOO, Belgrade Serbia, provided financial compensation to the experts from the given countries for participation at the advisory board meeting. Sanofi Bulgaria funded the medical writing and the journal fees. The project did not receive any additional grant or funding.

Medical Writing and/or Editorial Assistance. Gabor Kovacs, MD, the employee of Syreon Research Institute provided assistance with organizing the expert meeting and medical writing. Syreon Research Institute was the contracted partner of Sanofi Bulgaria.

Authorship. All named authors meet the International Committee of Medical Journal Editors (ICMJE) criteria for authorship for this article, take responsibility for the integrity of the work as a whole, and have given their approval for this version to be published.

Authorship Contributions. Nicolae Hancu: drafting the discussed topics, contribution to forming the expert opinion, facilitating the meeting discussion, leading the manuscript preparation, approving the final draft of the manuscript. Andrej Janez: contribution to forming the expert opinion, participation at meeting and the manuscript preparation, approving the final draft of the manuscript. Nebojsa Lalic: contribution to forming the expert opinion, participation at meeting and the manuscript preparation, approving the final draft of the manuscript. Nikolaos Papanas: contribution to forming the expert opinion, participation at meeting and the manuscript preparation, approving the final draft of the manuscript. Dario Rahelic: contribution to forming the expert opinion, participation at meeting and the manuscript preparation, approving the final draft of the manuscript. Gabriela Roman: contribution to forming the expert opinion, participation at meeting and the manuscript preparation, approving the final draft of the manuscript. Cristian Serafinceanu: contribution to forming the expert opinion, participation at meeting and the manuscript preparation, approving the final draft of the manuscript. Nikolaos Tentolouris: contribution to forming the expert opinion, participation at meeting and the manuscript preparation, approving the final draft of the manuscript. Blaženko Vukovic: contribution to forming the expert opinion, participation at meeting, leading the manuscript preparation, approving the final draft of the manuscript. Agron Ylli: contribution to forming the expert opinion, participation at meeting, leading the manuscript preparation, approving the final draft of the manuscript. Tsvetalina Tankova: contribution to forming the expert opinion, participation at meeting, leading the manuscript preparation, approving the final draft of the manuscript

Disclosures. Nicolae Hancu, Andrej Janez, Nebojsa Lalic, Papanas Nikolaos, Dario Rahelic, Gabriela Roman, Cristian Serafinceanu, Tsvetalina Tankova, Nikolaos Tentolouris, Blaženko Vukovic, Agron Ylli received remuneration for their participation to Advisory board organized by Sanofi but have received no honoraria for publication development apart from editorial support through a medical writing agency.

Compliance with Ethics Guidelines. This article is based on previously conducted studies, published guidelines and opinions of leading experts and does not contain any studies with 
human participants or animals performed by any of the authors.

Data Availability. Data sharing is not applicable to this article as no datasets were generated or analyzed during the current study.

Open Access. This article is licensed under a Creative Commons Attribution-NonCommercial 4.0 International License, which permits any non-commercial use, sharing, adaptation, distribution and reproduction in any medium or format, as long as you give appropriate credit to the original author(s) and the source, provide a link to the Creative Commons licence, and indicate if changes were made. The images or other third party material in this article are included in the article's Creative Commons licence, unless indicated otherwise in a credit line to the material. If material is not included in the article's Creative Commons licence and your intended use is not permitted by statutory regulation or exceeds the permitted use, you will need to obtain permission directly from the copyright holder. To view a copy of this licence, visit http://creativecommons. org/licenses/by-nc/4.0/.

\section{REFERENCES}

1. Davies MJ, D'Alessio DA, Fradkin J, et al. Management of hyperglycemia in type 2 diabetes, 2018. A consensus report by the American Diabetes Association (ADA) and the European Association for the Study of Diabetes (EASD). Diabetes Care. 2018;41(12):2669-701. https://doi.org/10.2337/ dci18-0033.

2. Khunti K, Giorgino F, Berard L, Mauricio D, Harris SB. The importance of the initial period of basal insulin titration in people with diabetes. Diabetes Obes Metab. 2020;22(5):722-33. https://doi.org/10. 1111/dom.13946.

3. Berard L, Bonnemaire M, Mical M, Edelman S. Insights into optimal basal insulin titration in type 2 diabetes: results of a quantitative survey. Diabetes Obes Metab. 2018;20(2):301-8. https:// doi.org/10.1111/dom.13064.

4. Perreault L, Vincent L, Neumiller JJ, SantosCavaiola T. Initiation and titration of basal insulin in primary care: barriers and practical solutions. J Am Board Family Med. 2019;32(3):431-47. https://doi.org/10.3122/jabfm.2019.03.180162.

5. Khunti S, Davies M, Khunti K. Clinical inertia in the management of type 2 diabetes mellitus: a focused literature review. Br J Diabetes Vasc Dis. 2015;15(2): 65-9.

6. Paul SK, Klein K, Thorsted BL, Wolden ML, Khunti $K$. Delay in treatment intensification increases the risks of cardiovascular events in patients with type 2 diabetes. Cardiovasc Diabetol. 2015;14:100. https://doi.org/10.1186/s12933-015-0260-x.

7. Khunti K, Gomes MB, Pocock S, et al. Therapeutic inertia in the treatment of hyperglycaemia in patients with type 2 diabetes: a systematic review. Diabetes Obes Metab. 2018;20(2):427-37. https:// doi.org/10.1111/dom.13088.

8. Lipska KJ, Ross JS, Miao Y, Shah ND, Lee SJ, Steinman MA. Potential overtreatment of diabetes mellitus in older adults with tight glycemic control. JAMA Intern Med. 2015;175(3):356-62. https://doi. org/10.1001/jamainternmed.2014.7345.

9. Aschner P, Gagliardino JJ, Ilkova H. Poor glycemic control in people with T1D and T2D: results from the International Diabetes Management Practices Study (IDMPS). ADA. 2018;1656-P.

10. Campbell MD, Babic D, Bolcina U, et al. High level of clinical inertia in insulin initiation in type 2 diabetes across Central and South-Eastern Europe: insights from SITIP study. Acta Diabetol. 2019;56(9):1045-9. https://doi.org/10.1007/ s00592-019-01346-1.

11. Lang BV, Markovic BB, Kranjcevic K. Family physician clinical inertia in glycemic control among patients with type 2 diabetes. Medl Sci Monitor. 2015;21:403-11. https://doi.org/10.12659/MSM. 892248.

12. Meneghini LF, Mauricio D, Orsi E, et al. The Diabetes Unmet Need with Basal Insulin Evaluation (DUNE) study in type 2 diabetes: achieving HbA1c targets with basal insulin in a real-world setting. Diabetes Obes Metab. 2019;21(6):1429-36. https:// doi.org/10.1111/dom.13673.

13. Mocarski M, Yeaw J, Divino V, et al. Slow titration and delayed intensification of basal insulin among patients with type 2 diabetes. J Managed Care Specialty Pharmacy. 2018;24(4):390-400. https://doi. org/10.18553/jmcp.2017.17218.

14. Freemantle N, Mauricio D, Giaccari A, et al. Realworld outcomes of treatment with insulin glargine $300 \mathrm{U} / \mathrm{mL}$ versus standard-of-care in people with uncontrolled type 2 diabetes mellitus. Curr Med Res 
Opin. 2020:36(4):571-81. https://doi.org/10.1080/ 03007995.2019 .1708287$.

15. Russell-Jones D, Dauchy A, Delgado E, et al. Take control: a randomized trial evaluating the efficacy and safety of self- versus physician-managed titration of insulin glargine $300 \mathrm{U} / \mathrm{mL}$ in patients with uncontrolled type 2 diabetes. Diabetes Obes Metab. 2019;21(7):1615-24. https://doi.org/10.1111/dom. 13697.

16. Berard L, Antonishyn N, Arcudi K, et al. Insulin matters: a practical approach to basal insulin management in type 2 diabetes. Diabetes Ther.
2018;9(2):501-19. https://doi.org/10.1007/s13300018-0375-7.

17. Little S, Shaw J, Home P. Hypoglycemia rates with basal insulin analogs. Diabetes Technol Ther. 2011;13(Suppl 1):S53-64. https://doi.org/10.1089/ dia.2011.0022.

18. Kalra S, Bajaj S, Sharma SK, et al. A practitioner's toolkit for insulin motivation in adults with type 1 and type 2 diabetes mellitus: evidence-based recommendations from an international expert panel. Diabetes Ther. 2020. https://doi.org/10.1007/ s13300-020-00764-7. 